\title{
Inter-Molecular Migration During Collisional Activation Monitored by Hydrogen/Deuterium Exchange FT-ICR Tandem Mass Spectrometry
}

\author{
Charlotte Hagman and Per Håkansson \\ Department of Engineering Sciences, Division of Ion Physics, Uppsala University, Uppsala, Sweden
}

Jos Buijs

Biacore AB, Uppsala, Sweden

Kristina Håkansson

Department of Chemistry, University of Michigan, Ann Arbor, Michigan, USA

\begin{abstract}
The difficulty with integrating solution-phase hydrogen/deuterium exchange (HDX) and tandem mass spectrometry is that the energy added to cause fragmentation might promote gas-phase migration of the added deuterium atoms. Here, we compare the solution-phase HDX profiles generated from $a$ - $b$ - and $y$-type fragment ion series originating from capillaryskimmer dissociation. The isotopic distributions of fragments from the different fragment ion types were used to determine the isotopic state of the amide hydrogen within a specific residue. Even though the same amide hydrogen was examined, the result was different for different fragment ion types. This observation indicates that different fragment series are not equally subjected to inter-molecular migration during collision-induced dissociation (CID). We also investigated the gas-phase reactivity of originally undeuterated CID fragments of penta-phenylalanine using gas-phase HDX in an external accumulation hexapole. The incorporation of deuterium into the different fragments was studied as a function of hexapole pressure. It was found that different $b$ - and $y$-ions from the same peptide had different gas-phase reactivity. However, the $a$-ions did not display significant gas-phase reactivity. The observed behavior has significant impact on any method that involves comparing the isotopic distributions of different fragment ions. Great care has to be taken in the interpretation of the HDX data using CID to increase the spatial resolution. The isotopic state observed after solution-phase exchange might be more preserved for some CID-fragment types. (J Am Soc Mass Spectrom 2004, 15, 639-646) (C) 2004 American Society for Mass Spectrometry
\end{abstract}

$\mathrm{T}$ The first study involving solution-phase hydrogen/deuterium exchange, HDX, in combination with mass spectrometry was published in 1991 by Katta and Chait [1]. That paper described the conformational changes in ubiquitin induced by changing the solvent composition. Mass spectrometry has several advantages compared with; it is less time consuming, more sensitive, and it can be used to study larger proteins. Over the last decade, the experimental approach of combining HDX and mass spectrometry has proven to be an excellent analytical tool and it has been used in a vast variety of studies. The standard approach for obtaining structural information with HDX and mass spectrometry involves three steps: First, the pro-

Published online February 27, 2004

Address reprint requests to Dr. C. Hagman, Department of Engineering Sciences, Division of Ion Physics, Angstrom Laboratory, Uppsala University, Box 534 S-751 21 Uppsala, Sweden. E-mail: Charlotte. Hagman@angstrom.uu.se tein is subjected to HDX for a certain time period. Second, the exchange is quenched by lowering the $\mathrm{pH}$ and the temperature. Third, a proteolytic enzyme, active under quenching conditions, is added. The protein digest is then analyzed with a mass spectrometer and the deuterium incorporation is examined. The proteolytic fragments are localised to different parts of the protein by peptide mapping [2-8].

Amide hydrogens participating in secondary structure, such as $\alpha$-helices and $\beta$-sheets, exchange slower than labile amides hydrogens accessible to the solvent with a factor of one thousand [9]. The exchange rate is dependent on factors, such as the $\mathrm{pH}$ and temperature of the surrounding solution [10]. The isotopic exchange of solvent-accessible amide hydrogens is also affected by the three-dimensional structure of the neighboring amino acid side chains: Bulky side chains make it more difficult for the solvent molecules to interact with the amide hydrogen, and the exchange will be slow [10]. The exchange rate dependency on the solvent accessi- 
bility of amide hydrogens can also be used to probe protein-protein interactions [11].

The standard HDX protocol provides medium resolution since it gives information about the solvent accessibility of an entire proteolytic fragment. To elucidate detailed biological processes, it is often important to determine more precisely where an interaction on the peptide backbone occurs. Therefore, there is a need for methods that can provide higher structural resolution.

In order to increase the spatial resolution, proteolytic digestion could be complemented or replaced with other fragmentation techniques, such as collision induced dissociation (CID) [12, 13].

Under low energy conditions, the dominant CID fragment types are $b$ - and $y$-ions, but energetic $b$-ions can be decomposed into $a$-ions by releasing a CO-group [14]. The mass difference between two consecutive fragments of the same fragment ion type identifies the additional amino acid residue of the larger fragment $[15,16]$. It is still a matter of debate whether amino acid residue specific information can be obtained with tandem mass spectrometry and isotopic labelling techniques. A major problem with the combination of HDX and physical fragmentation techniques, such as CID, is that gas-phase deuterium scrambling, which obscures information about solution-phase exchange processes, can occur as a result of the increased internal energy. Gas-phase HDX occurs through a relay mechanism and depends on the gas-phase acidities and proton affinities of the different constituents of the exchanging complex [17-19]. It is also clear that the collision energy is an important factor [20]. Earlier studies of deuterium scrambling indicate that the process is closely connected to the experimental set-up.

Johnson et al. investigated the proton mobility in low-energy collision induced fragmentation of small deuterated peptides in a triple quadrupole instrument and found that it resulted in gas-phase migration of the amide hydrogens [21]. McLafferty et al. investigated sustained off-resonance irradiation (SORI) CID of deuterated cytochrome $c$ in a Fourier transform ion cyclotron resonance (FT-ICR) instrument and reported extensive scrambling [22]. SORI-CID is a slow heating process, which increases the possibility of exchange processes occurring prior to peptide bond dissociation. Kaltashov et al. have proposed a model for HDX reactions in the gas phase [20]. This model includes complex formation between the reactant gas and a protonated site, and isotopic exchange within that complex. Following exchange, the complex is dissociating and intramolecular exchange can occur. The internal energy of the peptide ion of interest affects the different steps in the model. A high internal energy decreases the rate of formation of the exchanging complex, and results in less deuterium incorporation [20].

In contrast to the experiments described above, Anderegg et al. used tandem mass spectrometry in a triple quadrupole instrument to study short deuterated helical peptides and reported that scrambling was not significant during CID [23]. Also in other papers, the structural information obtained by HDX in combination with physical fragmentation methods is in agreement with information obtained by NMR: In a study by Deng et al., the average mass increase of consecutive CID fragments was determined after HPLC separation of peptic fragments of cytochrome $c$. They reported no scrambling for $b$-ions but found that $y$-ions were subjected to scrambling [24]. In order to elucidate the formation of fibrils, insulin has been studied by HDX and tandem MS during folded and aggregated conditions [25]. The study showed that an increased accessibility of the C-terminal region triggers the conversion from soluble to fibril form. These results are consistent with other studies concerning the conversion of different proteins to fibril form.

The most established method for obtaining structural information at high resolution is to measure the difference in the average deuterium incorporation between two consecutive peptide fragments. In order to more precisely determine the isotopic state of a particular amide hydrogen, the isotopic distributions of two consecutive fragments can be utilized, rather than the average mass increase of these fragments [13, 26]. However, only abundant fragments result in accurate calculations. Therefore only efficient fragmentation methods can be used for this type of analysis.

The approach of comparing either the average mass, or the isotopic distributions, of two peptide fragments is based on the assumption that the peptide fragments are non-reactive in the gas phase $[13,24-26]$. Consecutive $a$ or $b$-fragments should affect the distribution function (see eq 4 below) for the amide hydrogen equally and the rate for the exchange process at one specific residue should be the same in both cases. Here, we investigate these assumptions by both solution- and gas-phase HDX experiments. In the solution-phase exchange experiments the deuterium-to-hydrogen exchange (DHX) process at specific residues in small neuropeptides was monitored. The gas-phase experiments, involving CID fragments, were performed in an external accumulation hexapole [27].

\section{Experimental}

\section{Sample Preparation}

The peptides penta-phenylalanine (Phe)5, substance P(Arg-Pro-Lys-Pro-Gln-Gln-Phe-Phe-Gly-Leu-Met$\mathrm{OCH}_{3}$ ), and $\left[\mathrm{Tyr}^{4}\right]$-bombesin (pGlu-Gln-Arg-Tyr-GlyAsn-Gln-Trp-Ala-Val-Gly-His-Leu-Met- $\mathrm{NH}_{2}$ ) were purchased from Sigma Aldrich (Stockholm, Sweden). The peptides were fully deuterated by dissolving them in $\mathrm{D}_{2} \mathrm{O}\left(99.9 \%\right.$ Sigma Aldrich) to a concentration of $10^{-4}$ M. The (Phe) 5 sample had to be heated to $45^{\circ} \mathrm{C}$ and sonicated for $20 \mathrm{~min}$ in order to properly dissolve. The dissolved peptides were allowed to exchange for at least $24 \mathrm{~h}$. Deuterium to hydrogen back-exchange was 
initiated by mixing $30 \mu \mathrm{l}$ of the deuterated peptides with $390 \mu \mathrm{l}$ of a solution consisting of $50 \% \mathrm{H}_{2} \mathrm{O}, 47 \%$ methanol (Merck, Darmstad, Germany), and 3\% acetic acid (Sigma, Steinheim, Germany).

The final deuterium content relative to all exchangeable hydrogen isotopes in this mixture is $10 \%$. The peptide concentration was $10 \mu \mathrm{M}$ and the $\mathrm{pH}$ was 2.85-2.9 during all experiments. At this $\mathrm{pH}$, the amide hydrogen exchange rates are at their minima, allowing on-line monitoring of the exchange profiles. For all experiments no polymers could be observed in the mass spectra.

\section{HDX and Mass Spectrometry}

Solution-phase DHX experiments of peptides. Following dilution of deuterated peptides into spraying solvents (see above), peptide solutions were rapidly loaded into a $250 \mu \mathrm{l}$ syringe (Hamilton Co., Reno, NV), which was connected to an electrospray ion source (Analytica of Branford, Branford, CT). Nitrogen was used as nebulizing gas and a potential difference of $4 \mathrm{kV}$ was applied between the electrospray needle and the entrance capillary. The housing of the Analytica source was constantly purged with dry nitrogen to create a similar atmosphere for all experiments. Capillary-skimmer fragment ion spectra were acquired on a 9.4 tesla FT-ICR mass spectrometer (BioApex-94e, Bruker Daltonics, Billerica, MA). The capillary voltage was optimised to maximise the fragmentation efficiency for the different peptides. The following values were used: 350 $\mathrm{V}$ for (Phe)5, $350 \mathrm{~V}$ for substance $\mathrm{P}$, and $320 \mathrm{~V}$ for bombesin. Following dissociation, peptide fragment ions were externally accumulated in a hexapole for 2-2.5 s. Solution-phase exchange profiles were obtained by acquiring fragment ion spectra continuously during the exchange. All spectra represent an average of 65 scans.

Gas-phase HDX experiments of peptide fragments. A black dust coated capillary [28] with an i.d. of $50 \mu \mathrm{m}$ was coupled to a home-built pressure-directed infusion electrospray apparatus. The pressure inside the infusion pump was 0.3 bar during the experiments. A computercontrolled shutter was mounted in front of the entrance to the mass spectrometer [29]. A defined ion ensemble, determined by $t_{\text {injection, }}$ was fragmented in the capillaryskimmer area. Fragment ions were accumulated in the external hexapole and allowed to react with gaseous $\mathrm{D}_{2} \mathrm{O}$ for a certain time period, $\mathrm{t}_{\text {storage }}$. To avoid multiple storage assisted dissociation, MSAD, the $t_{\text {injection }}$ was set to $0.8 \mathrm{~s}$ and $t_{\text {storage }}$ was set to $1.0 \mathrm{~s}$. The hexapole pressure was raised from $\sim 1 \times 10^{-5}$ mbar to $\sim 5 \times 10^{-5}$ mbar by leaking in $\mathrm{D}_{2} \mathrm{O}$ through the liquid-gas inlet system of the mass spectrometer prior to the experiment. The capillary voltage was $350 \mathrm{~V}$ and each mass spectrum represents an average of 300 scans.

\section{Data Analysis}

Isotopic distributions. The distribution function of an element that has two possible states is a binomial distribution, if the element has three possible states it is a trinomial distribution. To calculate the probability, $\mathrm{f}$, that a certain number, $\mathrm{k}$, of atoms of one element are in a particular isotopic state of two possible (i.e., ${ }^{12} \mathrm{C}$ or ${ }^{13} \mathrm{C}$ ), a binomial distribution of the following form was used:

$$
f(k)=\left(\frac{n !}{k !(n-k) !}\right) p_{\text {elem }}{ }^{k}\left(1-p_{\text {elem }}\right)^{(n-k)}
$$

in which $n=$ the total number of atoms of that particular element, and $p_{\text {elem }}=$ the relative abundance of the particular isotope. The relative abundance of naturally occurring isotopes, $p_{\text {elem }}$ are known. For a molecule consisting of known numbers of atoms of particular elements, the isotopic distribution is given by a convolution of the binomial distributions of those elements. For a molecule built up from $\mathrm{C}, \mathrm{H}, \mathrm{N}$, and $\mathrm{O}$, the isotopic distribution of the molecule, $f_{m o l}(j)$, is given by eq 2.

$$
\begin{aligned}
f_{m o l}(j)= & \sum f_{C}^{n_{C}}(a) f_{H}^{n_{H}}(b) f_{N}^{n_{N}}(c) f_{O}^{n_{O}}(d), \\
& \text { for all } \mathrm{j}=\mathrm{a}+\mathrm{b}+\mathrm{c}+\mathrm{d}
\end{aligned}
$$

In the distribution function for the oxygen, $f_{O}^{n_{O}}(d)$, the $f_{O}$ provides information about which element is of interest, the $f^{n_{O}}$ tells the number of oxygen atoms present in the molecule and the (d), in which isotopic state the atom is. For a trinomial distribution the variable $\mathrm{d}$ can be either 0,1 or 2 . If hydrogen atoms bound to $S, O, N$, are in an environment which contains deuterium, incorporation will occur at the labile sites. Then, an additional distribution function for the labile hydrogens, $f_{H}^{n_{H}} /$ $D^{(e)}$, has to be added to eq 2 .

$$
\begin{aligned}
f_{\text {mol }}(i)= & \sum f_{C}^{n_{c}}(a) f_{N}^{n_{N}}(b) f_{O}^{n_{O}}(c) f_{H}^{n_{H}}(d) f_{H / D}^{n_{H}}(e), \\
& \text { for all } \mathrm{i}=\mathrm{a}+\mathrm{b}+\mathrm{c}+\mathrm{d}+\mathrm{e}
\end{aligned}
$$

To calculate the isotopic state of a particular amide hydrogen from two consecutive fragment ions of the same fragment type an equation of the following type was used:

$$
\begin{aligned}
A_{m+1}(k)= & A \operatorname{corr} \sum_{\mathrm{k}} A_{m}(q)\left(\operatorname{fres}(r) f_{H / D}(s)\right), \\
& \text { for all } \mathrm{k}=\mathrm{q}+\mathrm{r}+\mathrm{s}
\end{aligned}
$$

in which $\mathrm{A}_{(\mathrm{m}+1)}(\mathrm{k})$ corresponds to the relative magnitudes of all isotopic peaks, $\mathrm{k}$, observed for the larger fragment. $A_{(m)}(q)$ corresponds to the relative magnitudes of all isotopic peaks, $q$, observed for the smaller fragment. $f_{\text {res }}(r)$ corresponds to the theoretical relative abundances of the isotopic peaks, $r$, of the intermediate 
amino acid residue, $\mathrm{f}_{\mathrm{H} / \mathrm{D}}(\mathrm{s})$, corresponds to the relative abundance of hydrogen isotopes, $\mathrm{s}$, caused by the added deuterium and $\mathrm{A}_{\text {corr }}$ is a factor that corrects for the use of relative abundances instead of probabilities. The only unknown in eq 4 is the $\mathrm{f}_{\mathrm{H} / \mathrm{D}}(\mathrm{s})$ function, which was determined by a least-square analysis.

\section{Deuterium to Hydrogen Exchange Behavior at a Specific Amide Linkage}

The deuterium to hydrogen back exchange should follow the equation below:

$$
p_{\mathrm{D}}=(1-\varepsilon) e^{-k_{e x} t}+(\varepsilon)
$$

in which $\varepsilon$ is the $\mathrm{D} /(\mathrm{D}+\mathrm{H})$ ratio in solution, which amounts to 0.1 in the present experiments. The $\mathrm{D}$ to $\mathrm{H}$ exchange rate constant is given by $k_{\mathrm{ex}}$. At time zero, the probability of an amide hydrogen to be deuterated is equal to one. At equilibrium, the deuterium content at labile sites reflects the isotopic ratio in solution. Deviations from this behavior are consequences of gas-phase exchange processes.

\section{Results and Discussion}

In the solution-phase exchange experiments, the modified neuropeptide substance $\mathrm{P}-\mathrm{OCH}_{3}$ and $\left(\mathrm{Tyr}^{4}\right)$-bombesin were used. The size of peptides in a tryptic digest is in the same range.

Extensive fragmentation was observed following capillary-skimmer dissociation and both $b$ - and $a$-fragments were obtained from both peptides. The difference between the isotopic distribution function for the $b$-and $a$-fragments is that the $b$-fragments contain two additional components: the isotopic distribution function for the carbon and oxygen of the carbonyl group.

The magnitudes of the obtained $b$ and $a$-fragments allowed calculation of the isotopic state at specific residues in both substance $\mathrm{P}_{-} \mathrm{OCH}_{3}$ and $\left(\mathrm{Tyr}^{4}\right)$-bombesin. As an example, the deuterium level for three amide hydrogens are evaluated as function of the exchange time in solution. By extrapolating the generated exchange curve to time point zero and to infinity both the initial and final probabilities of having a deuterium at a particular amide hydrogen position were established. For a fully deuterated peptide, the initial deuterium content at labile amide sites is 1.0 and the equilibrium level of deuterium is 0.1 , reflecting the deuterium content in solution. The isotopic state of the amide hydrogen at the ninth residue, a glycine, in substance $\mathrm{P}-\mathrm{OCH}_{3}$ could be determined in two separate calculations using either $a_{8^{-}}, a_{9^{-}}$or $b_{8^{-}}, b_{9}$-fragments, Figure 1. The initial deuterium content, using the $a$ - and $b$-fragments, was 0.76 and 0.54 , respectively, instead of the expected 1.0. The equilibrium level was 0.25 and 0.24 using both fragment types instead of the expected

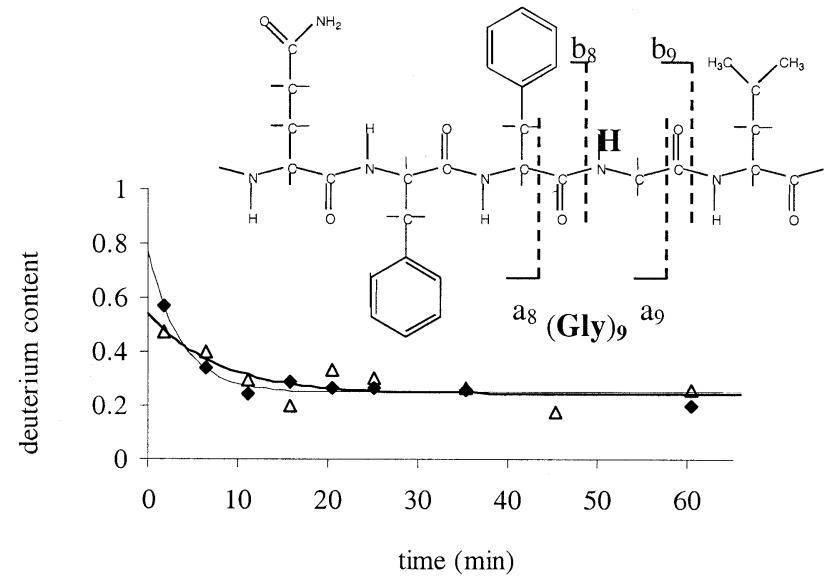

Figure 1. The $\mathrm{D} / \mathrm{H}$ exchange of the amide hydrogen at the ninth residue, a glycine residue, in substance $\mathrm{P}^{-} \mathrm{OCH}_{3}$ is plotted as a function of the reaction time in solution. The exchange behavior could be followed by two separate calculations since intense $a_{8}$, $a_{9^{-}}$, and $b_{8^{-}}, b_{9}$-fragment ions were obtained in the fragmentation process. The filled diamond represents the exchange behavior using the $a$-fragments in the calculations and the open triangle represent the behavior calculated from the $b$-fragments. During the experiment some gas-phase exchange occurred since the observed exchange behavior deviates from eq 5 . Even though the same amide hydrogen was studied the results from the different fragment series were not in perfect agreement.

0.1. The initial and final measured deuterium content did not reflect the deuterium content in solution.

An even more striking difference between the two fragment series was observed in the elucidation of the exchange processes of the amide hydrogen of the phenylalanine at the seventh residue in substance $\mathrm{P}-\mathrm{OCH}_{3}$, Figure 2. Basing the calculations on $a$-fragments resulted in an exchange process for the amide hydrogen with an initial deuterium content of 0.62 , which is lower than the expected 1.0 and a solution isotopic ratio equilibrium of 0.14 , which is higher than the expected 0.1 . The exchange process, as observed by using the $a$-fragments in the calculations, displayed exponentially decreasing exchange process whereas the results from the isotopic distributions of the $b$-fragments were centered at 0.2 , regardless of time.

The same observation was made in a study of the alanine amide hydrogen at the ninth residue in $\left(\mathrm{Tyr}^{4}\right)$ bombesin, Figure 3. The exchange process of the alanine amide hydrogen was investigated using the isotopic distributions of the $a_{9}$ and $a_{8}$-fragment.

Initially the measured deuterium content was 0.79 and the equilibrium level was 0.19 . The same exchange process could also be investigated using the corresponding $b$-fragments, $b_{9}$ and $b_{8}$. Then, the measured initial deuterium content of the amide hydrogen at the alanine residue was 0.42 and the equilibrium level was the same as for the $a$-fragments. From these results it is clear that gas-phase exchange reactions are taking place since the generated exchange curve is deviating from eq 5 . Again, using $a$-fragments in the calculations gave an exchange pattern which better reflects the exchange 


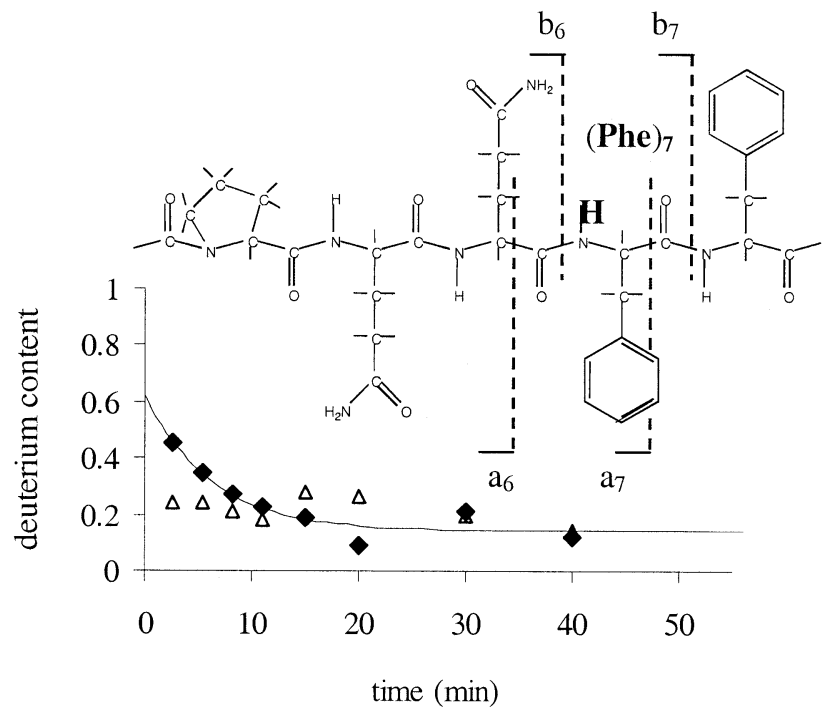

Figure 2. The $\mathrm{D} / \mathrm{H}$ exchange of the amide hydrogen at the seventh residue, a phenylalanine residue, in substance $\mathrm{P}_{-} \mathrm{OCH}_{3}$ is plotted as a function of the reaction time in solution. The isotopic state of the amide hydrogen was calculated by comparing the isotopic distributions of both the $a_{7}$ and $a_{6}$ or $b_{7}$ and $b_{6}$ fragments. The expected $\mathrm{D} / \mathrm{H}$ exchange behavior is more preserved and less scrambled using the isotopic distributions of the $a$-fragment ions, filled diamond, compared to the $b$-fragment ions, open triangle, in the calculations.

process in solution than basing the calculations on the isotopic distributions of the $b$-fragments. The explanation could be that the $a_{10^{-}}, a_{9^{-}}$, and $b_{10^{-}}, b_{9^{-}}$-fragments are not equally susceptible to gas-phase exchange during the experiment. The elevated levels found at infinite

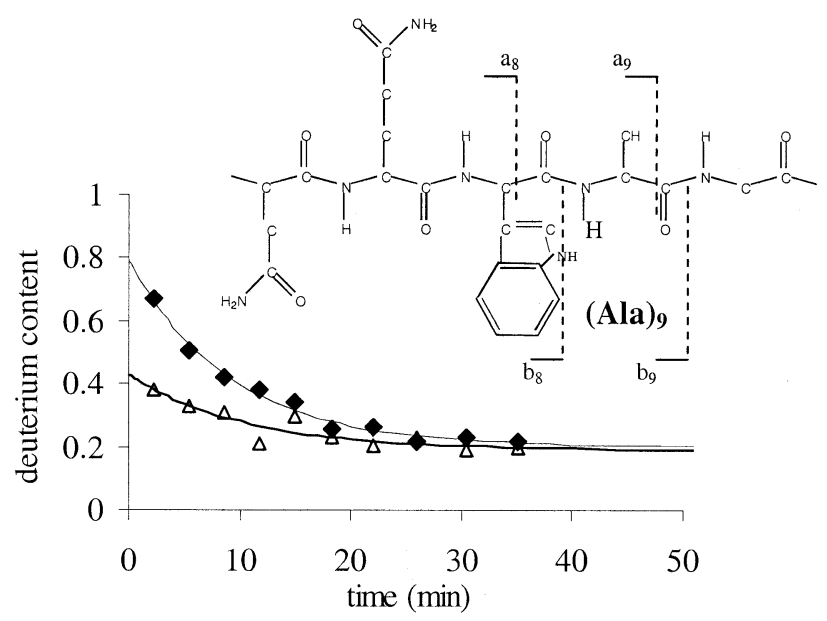

Figure 3. The $\mathrm{D} / \mathrm{H}$ exchange at the amide site at the ninth residue, an alanine residue, in $\left(\mathrm{Tyr}^{4}\right)$-bombesin is plotted as a function of the reaction time in solution. Both $b$ - and $a$-fragments were obtained from capillary-skimmer dissociation. The determination of the isotopic state of this hydrogen was made by using consecutive isotopic distributions from two fragment types in the calculations. The exchange process in solution is less scrambled using the $a$-fragments, filled diamond, instead of the $b$-fragments, open triangle. time could be explained if the $x_{n}$-fragment is more reactive in the gas-phase then the $\mathrm{x}_{n+1}$-fragment.

Previously, a similar deviation between the predicted isotopic state in solution and the measured isotopic state has been observed for amide hydrogens in a number of homopeptides [26].

\section{Gas-Phase Experiments}

To investigate the gas-phase reactivities of CID-fragments, an additional experimental approach was undertaken. The idea was to mimic the situation in the first pumping stage of the instrument during CIDconditions. An undeuterated peptide and its capillaryskimmer dissociation products were stored in an accumulation hexapole containing gaseous $\mathrm{D}_{2} \mathrm{O}$. The benefit of using an external ion accumulator, see Figure 4, instead of performing the gas-phase exchange reactions in the cell of the mass spectrometer is that there is no need for pump down periods before analysis can take place [27].

The gas-phase exchange involving $\mathrm{D}_{2} \mathrm{O}$ and dissociation fragments takes place through a simultaneous gain and loss of deuterium and hydrogen. The mechanism is referred to as the relay mechanism [17]. For gramicidin $\mathrm{S}$ it has been shown that at high internal energy there is a decrease in the extent of deuterium incorporation. That observation was explained by a large decrease in the formation rate and stability of the exchanging complex [20]. When using low-energy upfront collision-induced dissociation, the fragmentation takes place in the high pressure region of the mass spectrometer by applying a potential difference between the capillary and the skimmer. The peptide ions are accelerated and will collide with other molecules. The internal energy of the peptide ions is increasing after each collision and eventually a peptide bond, which is the weakest, will dissociate. The probability for gas-phase exchange is highest in the first pumping stage of the instrument due to the relatively high pressure.

In order to quantify the difference between the fragments' gas-phase reactivities, the relative mass increase for penta-phenylalanine fragments is plotted as a function of the reaction pressure, see Figure $5 a, b$, and c. This peptide has previously been shown to lose all information about its solution-phase exchange behavior during tandem mass spectrometric analysis [26]. For this peptide, the observed $a$-ion (shown in Figure 5a) and two of three observed $y$-ions (shown in Figure 5c) showed a linear increase of mass as a function of hexapole pressure. The obtained $b$-ions were more reactive and incorporated deuterium in a logarithmic manner, see Figure $5 \mathrm{~b}$. This observation is in contrast to what has been reported in another study concerning CID MS/MS and HDX. That study reported the gasphase exchange in both protonated and sodiated $a-, b-$, and $y$-ions and found similar gas-phase exchange prop- 


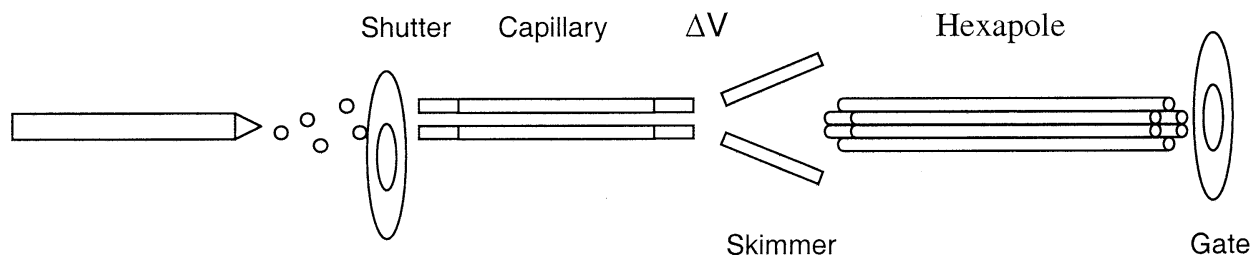

Figure 4. Gas-phase experiments were performed with originally undeuterated penta-phenyalanine, (Phe)5. The parent ion was fragmented with capillary-skimmer dissociation; both $a-, b-$, and $y$-fragments were observed. The peptide ions were accumulated in a hexapole, to which gaseous $\mathrm{D}_{2} \mathrm{O}$ had been added. The hexapole pressure was varied between $1 \times 10^{-5} \mathrm{mbar}$ and $5 \times 10^{-5} \mathrm{mbar}$. A shutter was mounted in front of the entrance to the mass spectrometer in order to control the amount of ions accumulated in the hexapole. The reaction time was fixed for all reaction pressures.

erties among different fragment types containing the same charge carrier [30].

A possible explanation to our findings is linked to the peptide dissociation process. It is known that the $b$-ions are protonated oxazalones [14]. The dissociation of $b_{2}$-ions into $a_{2}$-ions is accomplished by releasing the carbonyl carbon and oxygen along with a kinetic energy release. $\mathrm{Ab}$ initio calculations showed that the protonated oxazalone has the lowest energy structure and the resulting $\mathrm{a}_{2}$-ions and cabon monoxide have a potential energy of $0.61 \mathrm{eV}$ higher compared to the protonated oxazalone [14]. Studies of larger peptides showed that the fragmentation $\mathrm{b}_{n} \rightarrow \mathrm{a}_{n}+\mathrm{CO}(n=3,4,5)$ is linked to a large kinetic energy release and the products have higher free energy compared with the reactants [31]. In a study examining the gas-phase exchange dependence on parameters such as the reactant gas pressure and the degree of internal excitation of the reactant ions, a model for the isotopic exchange was presented [20]. It was suggested that the stability of the exchanging gas-phase complex is dependent on the internal energy of the peptide ions. If the peptide ion is highly energetic, the exchanging complex will be unstable and isotopic exchange will be less likely. The higher internal energy, compared with the $b$-fragments, of the produced $a$-fragments might lower the formation probability of the exchanging gas-phase complex according to the gas-phase exchange mechanism described above. Since the corresponding $b$-fragments are less energetic, the corresponding exchanging complex is more stable and therefore results in a higher degree of isotopic exchange.

In another study the activation energies in the collision induced dissociation process were investigated for smaller peptides. It was found that the activation energy for the $a_{1}$-fragment formation was both experimentally and theoretically close to the values suggested by Harrison et al. [14]. The activation energy for the $y_{1}$-fragment formation was somewhat lower compared to the $a_{1}$-fragment formation. The dissociation leading to $b_{2}$-fragment formation had the lowest activation energy [32]. In our experiments, the gas-phase reactivity of the $y$-fragments displayed a regular characteristic correlated to the size of the fragment. The smallest $y$-fragment incorporated most deuterium in the gas phase, resembling the typical $b$-fragment uptake. The larger fragments, $y_{3}$ and $y_{4}$, incorporated less deuterium and had a similar uptake profile as the $a_{4}$-fragment.

It has also been observed that the gas-phase exchange rate and extent have a close correlation to the gas-phase structure [33, 34]. Thus, another possible explanation for our experimental results could be that the different fragment types produced by CID have specific structures. The $b$-fragments have an oxazalone structure whereas the corresponding $y$-fragments have a linear configuration. The $a$-fragments are immonium ions formed through the loss of a CO-group from the $b$-ion oxazalone structure. The $a_{4}$-fragment and the two larger $y$-fragments displayed a similar gas-phase exchange behavior, both in terms of rate and extent, see Figure 5a and c. An explanation could be that these CID fragments adopt a rather compact gas-phase conformation which prevents gas-phase exchange. It is possible that the shortest $y$-fragment, the $y_{2}$-fragment, cannot adopt such a conformation in the gas phase due to its smaller size. In comparison with the other CID-fragment types, all three $b$-fragments displayed a different kinetics for the incorporation of deuterium, see Figure $5 b$. Consequently, the $b$-fragments had a conformation which is more favorable for gas-phase exchange reactions. The gas-phase reactivity of the fragment ions from bradykinin has been investigated by Mao and Douglas using a linear quadrupole ion trap [35]. In that experiment, the $y$-fragments were more reactive in the gas phase and incorporated most deuterium. It was not possible to find a consistent gas-phase characteristic for the $b$ - and $a$-fragments. This observation could be explained by the fact that the different amino acids in the peptide might cause consecutive fragments of the same type to display different gas-phase exchange properties due to different gas-phase conformations. Our results originate from CID-fragments of penta-phenylalanine and are thus independent of variations in amino acid composition.

\section{Conclusions}

Using the HDX profiles of consecutive peptide fragment ions to obtain amino acid residue-specific structural information is complicated because of the fact that 

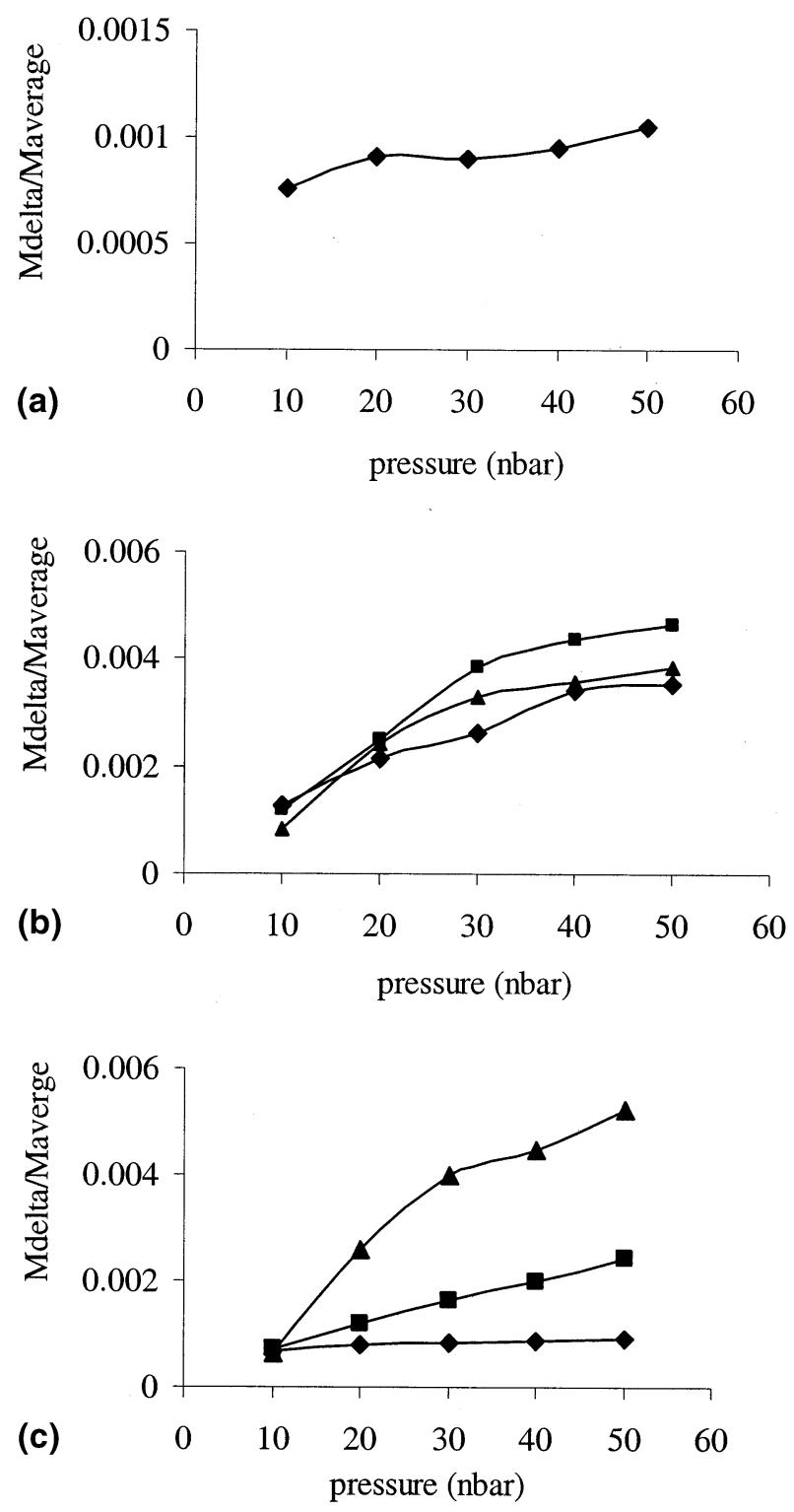

Figure 5. The relative mass increase of different fragment types and parent ion of penta-phenyalanine, (Phe)5, is plotted as a function of the reaction pressure in the accumulation hexapole. Prior capillary-skimmer dissociation resulted in $a_{4^{-}}, b_{2^{-}}, b_{3^{-}}, b_{4^{-}}, y_{2^{-}}$, $y_{3^{-}}$, and $y_{4}$-fragments at all reaction pressures. The relative deuterium incorporation into the $a_{4}$ fragment can be seen in (a), the incorporation into the $b$-fragments in (b), filled diamond $=b_{4}$, filled square $=b_{3}$, filled triangle $=b_{2}$, and the incorporation into the $y$-fragments, filled diamond $=y_{4}$, filled square $=y_{3}$, filled triangle $=y_{2}$, in (c).

different fragment types display different gas-phase exchange properties.

For penta-phenylalanine, a difference in gas-phase deuterium incorporation was observed between the generated fragment types and lengths. The variation could either be explained by different internal energy of the obtained fragments or different gas-phase conformations. Larger $y$ - and $a$-fragments are less reactive than $b$ - and $y_{2}$-fragments.

The differences in gas-phase reactivity of the CID fragments and between different fragment types have implications for how to interpret MS/MS data from solution-phase exchange experiments. In such experiments the same amide hydrogen can be examined by using different fragment types. Using the less gas-phase reactive fragment type will preserve more of the information about the solution-phase exchange process at specific amino acid positions. Alternatively, fragmentation techniques that occur in the low pressure region of the mass spectrometer should be used in HDX experiments.

\section{Acknowledgments}

The authors acknowledge Johan Kjellberg for technical assistance. This work was supported by the Knut and Alice Wallenberg Foundation and the Swedish Natural Science Research Council (NFR). JB acknowledges the Swedish KK-Foundation.

\section{References}

1. Katta, V.; Chait, B. T. Conformational Changes in Proteins Probed by Hydrogen-Exchange Electrospray-Ionization Mass Spectrometry. Rapid Commun. Mass Spectrom 1991, 5, 214-217.

2. Miranker, A.; Robinson, C. V.; Aplin, R. T.; Dobson, C. M. Detection of Transient Protein Folding Populations by Mass Spectrometry. Science 1993, 262, 896-900.

3. Zhang, Z.; Smith, D. L. Determination of Amide Hydrogen Exchange by Mass Spectrometry: A New Tool for Protein Structure Elucidation. Protein Sci 1993, 2, 522-531.

4. Smith, D. L.; Deng, Y.; Zhang, Z. Probing the Noncovalent Structure of Proteins by Amide Hydrogen Exchange and Mass Spectrometry. J. Mass Spectrom 1997, 32, 135-146.

5. Deng, Y.; Smith, D. L. Rate and Equilibrium Constants for Protein Unfolding and Refolding Determined by Hydrogen Exchange-Mass Spectrometry. Anal. Biochem 1999, 276, 150160.

6. Zhang, Z.; Li, W.; Logan, T. M.; Li, M.; Marshall, A. G. Human Recombinant [C22A] FK506-Binding Protein Amide Hydrogen Exchange Rates from Mass Spectrometry Match and Extend those from NMR. Protein Sci 1997, 10, 2203-2217.

7. Wang, F.; Li, W.; Emmett, M. R.; Hendrickson, C. L.; Marshall, A. G. Conformational and Dynamic Changes of Yersinia Protein Tyrosine Phosphatase Induced by Ligand Binding and Active Site Mutation and Revealed by H/D Exchange and Electrospray Ionization Fourier Transform Ion Cyclotron Resonance Mass Spectrometry. Biochemistry 1998, 37, 15289-15299.

8. Buijs, J.; Ramstrom, M.; Danfelter, M.; Larsericsdotter, H.; Håkansson, P.; Oscarsson, S. Localized Changes in the Structural Stability of Myoglobin upon Adsorption onto Silica Particles, as Studied with Hydrogen/Deuterium Exchange Mass Spectrometry. J. Colloid Interface Sci 2003, 263, 441-448.

9. Bay, Y.; Milne, J. S.; Mayne, L.; Englander, S. W. Protein Stability Parameters Measured by Hydrogen Exchange. Proteins 1994, 20, 4-14.

10. Bai, Y.; Milne, J. S.; Mayne, L.; Englander, S. W. Primary Structure Effects on Peptide Group Hydrogen Exchange. Proteins 1993, 17, 75-86.

11. Mandell, J. G.; Falick, A. M.; Komirevs, E. A. Measurment of Amid Hydrogen Exchange by MALDI-TOF Mass Spectrometry. Anal. Chem 1998, 70, 3987-3995.

12. Deng, Y.; Pan, H.; Smith, D. L. Selective Isotope Labeling Demonstrates that Hydrogen Exchange at Individual Peptide Amide Linkages Can Be Determined by Collision-Induced 
Dissociation Mass Spectrometry. J. Am. Chem. Soc 1999, 121, 1966-1967.

13. Buijs, J.; Håkansson, K.; Hagman, C.; Håkansson, P.; Oscarsson, S. A New Method for the Accurate determination of the Isotopic State of Single Amide Hydrogen within Peptides Using Fourier Transform Ion Cyclotron Resonance Mass Spectrometry. Rapid Commun. Mass Spectrom 2000, 14, 1751-1756.

14. Yalcin, T.; Khouw, C.; Csizmadia, I. G.; Peterson, M. R.; Harrison, A. G. Why are B Ions Stable Species in Peptide Spectra? J. Am. Soc. Mass Spectrom 1995, 6, 1165-1174.

15. Hunt, D. F.; Yates, J. R., III; Shabanowitz, J.; Winston, S.; Hauer, C. R. Protein Sequencing by Tandem Mass Spectrometry. Proc. Natl. Acad. Sci. U.S.A 1986, 83, 6233-6237.

16. Biemann, K.; Scoble, H. A. Characterization by Tandem Mass Spectrometry of Structural Modifications in Proteins. Science 1987, 237, 992-998.

17. Campbell, S.; Rodgers, M. T.; Marzluff, E. M.; Beauchamp, J. L. Deuterium Exchange Reactions as a Probe of Biomolecule Structure. Fundamental Studies of Gas Phase H/D Exchange Reactions of Protonated Glycine Oligomers with D2O, CD3OD, CD3CO2D, and ND3. J. Am. Chem. Soc 1995, 117, 12840-12854.

18. Wyttenbach, T.; Bowers, M. T. Gas Phase Conformations of Biological Molecules: The Hydrogen/Deuterium Exchange Mechanism. J. Am. Soc. Mass Spectrom 1999, 10, 9-14.

19. Schaaff, T. G.; Stephenson, J. L.; McLucky, S. A. Gas Phase $\mathrm{H} / \mathrm{D}$ Exchange Kinetics: DI versus $\mathrm{D}_{2} \mathrm{O}$. J. Am. Soc. Mass Spectrom 2000, 11, 167-171.

20. Kaltashov, I. A.; Doroshenko, V. M.; Cotter, R. J. Gas Phase Hydrogen/Deuterium Exchange Reactions of Peptide Ions in a Quadrupole Ion Trap Mass Spectrometer. Proteins 1997, 28, 53-58.

21. Johnson, R. S.; Krylov, D.; Walsh, K. A. Proton Mobility Within Electrosprayed Peptide Ions. J. Mass Spectrom 1995, 30, 386-387.

22. McLafferty, F. W.; Guan, Z.; Haupts, U.; Wood, T. D.; Kelleher, N. L. Gaseous Conformational Structures of Cytochrome c. J. Am. Chem. Soc 1998, 120, 4732-4740.

23. Anderegg, R. J.; Wagner, D. S.; Stevenson, C. L.; Borchardt, R. T. The Mass Spectrometry of Helical Unfolding in Peptides. J. Am. Soc. Mass Spectrom 1994, 5, 425-433.

24. Deng, Y. Z.; Pan, H.; Smith, D. L. Selective Isotope Labeling Demonstrates That Hydrogen Exchange at Individual Amide Linkages Can be Determined by Collision-Induced Dissociation Mass Spectrometry. J. Am. Chem. Soc 1999, 121, 1966-1967.
25. Tito, P.; Nettleton, E. J.; Robinson, C. V. Dissecting the Hydrogen Exchange Properties of Insulin Under Amyloid Fibril Forming Conditions: A Site-Specific Investigation by Mass Spectrometry. J. Mol. Biol 2000, 303, 267-278.

26. Buijs, J.; Hagman, C.; Håkansson, K.; Richter, J. H.; Håkansson, P. Inter- and Intra-Molecular Migration of Peptide Amid Hydrogens During Electrospray Ionization. J. Am. Soc. Mass Spectrom 2001, 12, 410-419.

27. Hofstadler, S. A.; Sannes-Lowery, K. A.; Griffey, R. H. A Gated-Beam Electrospray Ionization Source with an External Ion Reservoir. A New Tool for the Characterization of Biomolecules using Electrospray Ionization Mass Spectrometry. Rapid Commun. Mass Spectrom 1999, 13, 1971-1979.

28. Nilsson, S.; Wetterhall, M.; Bergquist, J.; Nyholm, L.; Markides, K. A Simple and Robust Conductive Graphite Coating for Sheathless Electrospray Emitters Used in Capillary Electrophoresis/Mass Spectrometry. Rapid Commun. Mass Spectrom 2001, 14, 1997-2000.

29. Axelsson, J.; Håkansson, K.; Palmblad, M.; Håkansson, P. A Method to Significantly Lessen the Sample Contamination of the Vacuum Interface of an On-Axis Electrospray Ion Source by Adding a Mechanical Shutter. Rapid Commun. Mass Spectrom 1999, 13, 1550.

30. Demmers, J. A.; Rijkers, D. T.; Haverkamp, J.; Killian, J. A.; Heck, A. J. Factors Affecting Gas-Phase Deuterium Scrambling in Peptide Ions and Their Implications for Protein Structure Determination. J. Am. Chem. Soc 2002, 124, 11191-11198.

31. Yalcin, T.; Csizmadia, I. G.; Peterson, M. R.; Harrison, A. G. The Structure and Fragmentation of $\mathrm{B}_{n}(n \geq 3)$ Ions in Peptide Spectra. J. Am. Soc. Mass Spectrom 1996, 7, 233-242.

32. Klassen, J. S.; Kebarle, P. Collision-Induced Dissociation Threshold Energies of Protonated Glycine, Glycinamide, and Some Related Small Peptides and Peptide Amino Amides. J. Am. Chem. Soc 1997, 119, 6552-6563.

33. Freitas, M. A.; Hendrickson, C. L.; Emmett, M. R.; Marshall, A. G. High-Field Fourier Transform Ion Cyclotron Resonance Mass Spectrometry for Simultaneous Trapping and Gas-Phase Hydrogen/Deuterium Exchange of Peptide Ions. J. Am. Soc. Mass Spectrom 1998, 9, 1012-1019.

34. Freitas, M. A.; Marshall, A. G. Rate and Extent of Gas-Phase Hydrogen/Deuterium Exchange of Bradykinins: Evidence for Peptide Zwitterions in the Gas Phase. Int. J. Mass Spectrom 1999, 182/183, 221-231.

35. Mao, D.; Douglas, D. J. H/D Exchange of Gas Phase Bradykinin Ions in a Linear Quadrupole Ion Trap. J. Am. Soc. Mass Spectrom 2003, 14, 85-94. 